Recepción: 20 / 01 / 2019

Aceptación: 19 / 03 / 2019

(c)

Ciencias de la salud

Publicación: 05 / 05 / 2019

Artículo de Revisión

\title{
Seguridad clínica hospitalaria: un desafío para los profesionales de salud y pacientes
}

\section{Hospital clinical safety: a challenge for health professionals and patients}

\section{Segurança clínica hospitalar: um desafio para profissionais de saúde e pacientes}

Cristhian P. Triviño-Ibarra ${ }^{\mathrm{I}}$

kristrivi6535@hotmail.com

Hernán D. Toro-Barrera ${ }^{\mathrm{II}}$ hdavidtorob@gmail.com

Hugo J. Saltos-Giler ${ }^{\text {III }}$ javilifer@hotmail.com

Julio C. Cedeño-Quijije IV jucecequi@hotmail.com

María D. Párraga-Zambrano $\mathrm{V}$ dioselina_34@hotmail.com

Mercedes M. Vicuña-Castro VI mercy09092010@hotmail.com

Correspondencia: kristrivi6535@hotmail.com

I. Licenciado en Enfermería; Maestrante en Seguridad Clínica del Paciente y Calidad de la Atención Sanitaria; Centro de Salud tipo C; Manta, Ecuador.

II. Médico Cirujano; Médico Residente de UCI Clínica Centeno; Médico Residente de Emergencia y Jefe de Guardia Hospital General IESS; Manta, Ecuador.

III. Médico Cirujano; IEES; Manta, Ecuador.

IV. Médico Cirujano; Médico Residente de Emergencia y Jefe de Guardia Hospital General IESS; Manta, Ecuador.

v. Médico Cirujano; Médico de Ministerio de Salud Pública de Ecuador; Centro de Salud de Guangaje Distrito 05D04; Cotopaxi, Ecuador.

VI. Licenciada en Enfermería; Magister en Dirección y Gestión Sanitaria; Hospital General IESS; Portoviejo, Ecuador. 
Cristhian P. Triviño-Ibarra; Hernán D. Toro-Barrera; Hugo J. Saltos-Giler; Julio C. Cedeño-Quijije; María D. PárragaZambrano; Mercedes M. Vicuña-Castro

\title{
Resumen
}

Una de las características que deben poseer toda organización es la seguridad y el ambiente laboral en la cual se desenvuelven sus empleados. Es por ello que la búsqueda de una cultura de seguridad hace incrementar una calidad de la misma. Aunado a esto si se aplica estándares internacionales a través de sistemas de gestión incrementará el bienestar de los trabajadores. Los centros hospitalarios no escapan de esta realidad y más porque hay presencia de riesgos que pueden poner en peligro la salud y el bienestar de los profesionales de la salud y pacientes. El control de la seguridad y la calidad de la misma se manifiesta a través de una excelente atención a los pacientes que acuden a estos centros asistenciales. Es por ello, que el objetivo general de esta investigación es analizar la importancia de la seguridad clínica hospitalaria para los profesionales y pacientes. La metodología utilizada se basó en investigaciones documentales y bibliográficas. Los resultaron que se obtuvieron se reflejaron a través de los análisis de los servicios de salud, la calidad de los mismos y el riesgo que pueden padecer los que forman parte de estos lugares. Como conclusión se determinó que la seguridad de los centros hospitalarios dependerá del conocimiento e identificación de los riesgos que puedan padecer los profesionales y pacientes que están dentro de estos sitios asistenciales, por lo que controlar los errores y fallos que puedan suceder repercute directamente en la disminución de los riesgos ya que habrá pocos efectos adversos para ello.

Palabras claves: Seguridad; Calidad; Riesgos; Efectos Adversos.

\begin{abstract}
One of the characteristics that every organization must possess is the safety and the working environment in which its employees operate. That is why the search for a safety culture increases the quality of it. Coupled with this if international standards are applied through management systems will increase the welfare of workers. The hospitals do not escape from this reality and more because there are risks that may endanger the health and well-being of health professionals and patients. The control of safety and the quality of it is manifested through excellent care to patients who come to these care centers. That is why, the general objective of this research is to analyze the importance of hospital clinical safety for professionals and patients. The methodology used was based on documentary and bibliographic research. The results that were obtained were reflected through the analysis of the health services, the quality of them and the risk that may be suffered by those who are part of these places. In conclusion, it was determined that the safety of the hospital centers will
\end{abstract}


depend on the knowledge and identification of the risks that professionals and patients that are inside these care sites may suffer, so controlling the errors and failures that may occur has a direct impact on the decrease of the risks since there will be few adverse effects for this.

Keys words: Security; Quality; Risks Adverse effects.

\section{Resumo.}

Uma das características que toda organização deve possuir é a segurança e o ambiente de trabalho em que seus funcionários operam. É por isso que a busca por uma cultura de segurança aumenta a qualidade dela. Juntamente com isso, se os padrões internacionais forem aplicados por meio de sistemas de gerenciamento, isso aumentará o bem-estar dos trabalhadores. Os hospitais não escapam dessa realidade e mais porque existem riscos que podem comprometer a saúde e o bem-estar dos profissionais de saúde e dos pacientes. O controle da segurança e a qualidade do mesmo se manifesta por meio de excelente atendimento aos pacientes que chegam a esses centros de atendimento. Por isso, o objetivo geral desta pesquisa é analisar a importância da segurança clínica hospitalar para profissionais e pacientes. A metodologia utilizada foi baseada em pesquisa documental e bibliográfica. Os resultados obtidos foram refletidos através da análise dos serviços de saúde, da qualidade dos mesmos e do risco que pode ser sofrido por aqueles que fazem parte desses locais. Em conclusão, determinou-se que a segurança dos centros hospitalares dependerá do conhecimento e identificação dos riscos que os profissionais e pacientes que estão dentro desses locais de cuidado podem sofrer, assim, controlar os erros e falhas que podem ocorrer tem um impacto direto sobre a diminuição dos riscos, uma vez que haverá poucos efeitos adversos para isso.

Palavras chaves: Segurança; Qualidade Riscos Efeitos adversos.

\section{Introducción.}

Las acciones de trabajo permiten que los trabajadores hagan un esfuerzo físico y psicológico de realizar un evento en pro de un colectivo o de manera individual. Estas acciones conllevan a que el individuo pueda estar en contacto con un sistema de riesgo y eventualmente en peligro por lo cual lo conlleva a sufrir algún accidente. Uno de los parámetros para evitar estos factores de riesgo es la experiencia de la persona en el puesto de trabajo como también el conocimiento que tenga de normas de seguridad laboral. 
Cristhian P. Triviño-Ibarra; Hernán D. Toro-Barrera; Hugo J. Saltos-Giler; Julio C. Cedeño-Quijije; María D. PárragaZambrano; Mercedes M. Vicuña-Castro

"Las condiciones de trabajo susceptibles de ocasionar alteraciones en la salud de la población laboral no es una exclusividad del sector industrial. Por ello, en la época actual, antes que hablar de "higiene y seguridad industrial", es mejor hablar de "seguridad laboral" o "seguridad en el trabajo". La "higiene" es consustancial a la seguridad. Esa separación ancestral entre "higiene" y "seguridad" también es inconsistente”.(Betancourt, 1999, pág. 56)

Esta seguridad laboral no escapa de lugares como los centros de salud, donde el riesgo de padecer alguna enfermedad es grande debido a los diferentes patógenos encontrados allí. Es por ello que la seguridad laboral en estos ámbitos es de suma importancia tanto para los profesionales como para los pacientes. Los pacientes son más vulnerables a sufrir estos tipos de eventos. Entre un 4 y un 17\% de los pacientes que ingresan en hospital sufrirán un accidente imprevisto e inesperado, derivado de la atención sanitaria y no de su enfermedad de base, que tendrá consecuencias en su salud y en sus posibilidades de recuperación(Revuelta \& Mingorance, 2011).

La importancia de la seguridad del paciente manifiesta la importancia que tiene la calidad de servicio dentro de estos servicios hospitalarios. La seguridad del paciente constituye un importante indicador de la calidad asistencial, y su mejora representa una prioridad para los servicios sanitarios por lo cual es esencial promover, crear y mantener una cultura de seguridad positiva en las organizaciones(Giménez-Júlveza, Hernández-García, Aibar-Remón, Gutiérrez-Cíac，\& FebrelBordejéd, 2017).

"La seguridad de los pacientes constituye un proceso complejo de implicancias subjetivas, individuales y colectivas, ético-políticas, científicas, prácticas y organizacionales que interviene de manera multidimensional e interdependiente en la atención de la salud en cuanto ella está vinculada con la promoción y protección de la vida, los derechos fundamentales de las personas, la provisión de ambientes y prácticas de salud libres de amenazas y riesgos, y la mitigación de los daños producidos por el proceso de atención”.(MSP, 2015, págs. 14-15)

Ahora, la seguridad que deben tener los profesionales dentro de los centros de salud está involucrado con el manejo de los residuos hospitalarios. Estos son considerados potencialmente peligrosos, ya sea por el riesgo de contaminación biológica con microorganismos patógenos, o química, por sustancias como drogas, carcinógenos y material radioactivo que pueden contener(Quinto-Mosquera, Pérez, \& Arias, 2013). 
La manipulación de estos desechos incrementa el riesgo para el trabajador hospitalario, que puede contaminarse la piel o las conjuntivas oculares, herirse con objetos cortopunzantes, inhalar aerosoles infectados o irritantes, o ingerir en forma directa o indirecta, el material contaminado(Natura \& Zabala, 1997). De aquí es necesario poder obtener un modelo de manejo de residuos hospitalarios con el fin de que el personal tenga conocimiento y a su vez no ocurran accidentes laborales por el alto riesgo que generan. Esto se puede observar en la Figura 1.

Es por ello, que todos los actores que estén involucrados en el bienestar y progreso del paciente deben estar atentos a estos eventos circunstanciales. El sistema de salud requiere que todos los responsables en la atención de la población, se involucren con las acciones destinadas a mejorar la calidad del servicio en los diversos ámbitos(León Román, 2006).

Los involucrados deben tener algún conocimiento o información de la seguridad laboral, es decir hay una relación entre medicina y seguridad. El conocimiento adecuado de las condiciones de trabajo permite inferir sobre los problemas de salud que puede sufrir la población laboral; además, no es factible conseguir mejores niveles de salud si no se interviene en las condiciones de trabajo(Betancourt, 1999).

La seguridad debe ser parte fundamental del desarrollo de las actividades dentro de los centros de salud. De aquí nace entonces una nueva cultura dentro de estos espacios hospitalarios. La cultura de seguridad es un conjunto de valores, actitudes, percepciones, competencias y objetivos, tanto individuales como de grupo, que determinan el grado de compromiso de las organizaciones para disminuir los riesgos y daños(Zula Majín, 2015).

Poder controlar esta seguridad requiere de mecanismos que tienen cierta complejidad ya que muchos pueden ser objetivos y subjetivos, dependiendo de quién las esté tomando en consideración. Esto afecta directamente a la atención sanitaria. Los procesos asistenciales suponen una combinación de actuaciones, tecnologías e interacciones humanas de un gran número de profesionales, por lo que se asocia a un riesgo creciente de efectos adversos y perjuicio involuntario para el paciente(Muiño Míguez, y otros, 2007).Algunos autores plantean que el error médico es el más importante factor causal de eventos adversos o consecuencias indeseadas del proceso de atención médica, muy por encima de la mala práctica o las condiciones del paciente, por lo que requiere una mayor vigilancia(Ramos Domínguez, 2005). 
Cristhian P. Triviño-Ibarra; Hernán D. Toro-Barrera; Hugo J. Saltos-Giler; Julio C. Cedeño-Quijije; María D. PárragaZambrano; Mercedes M. Vicuña-Castro

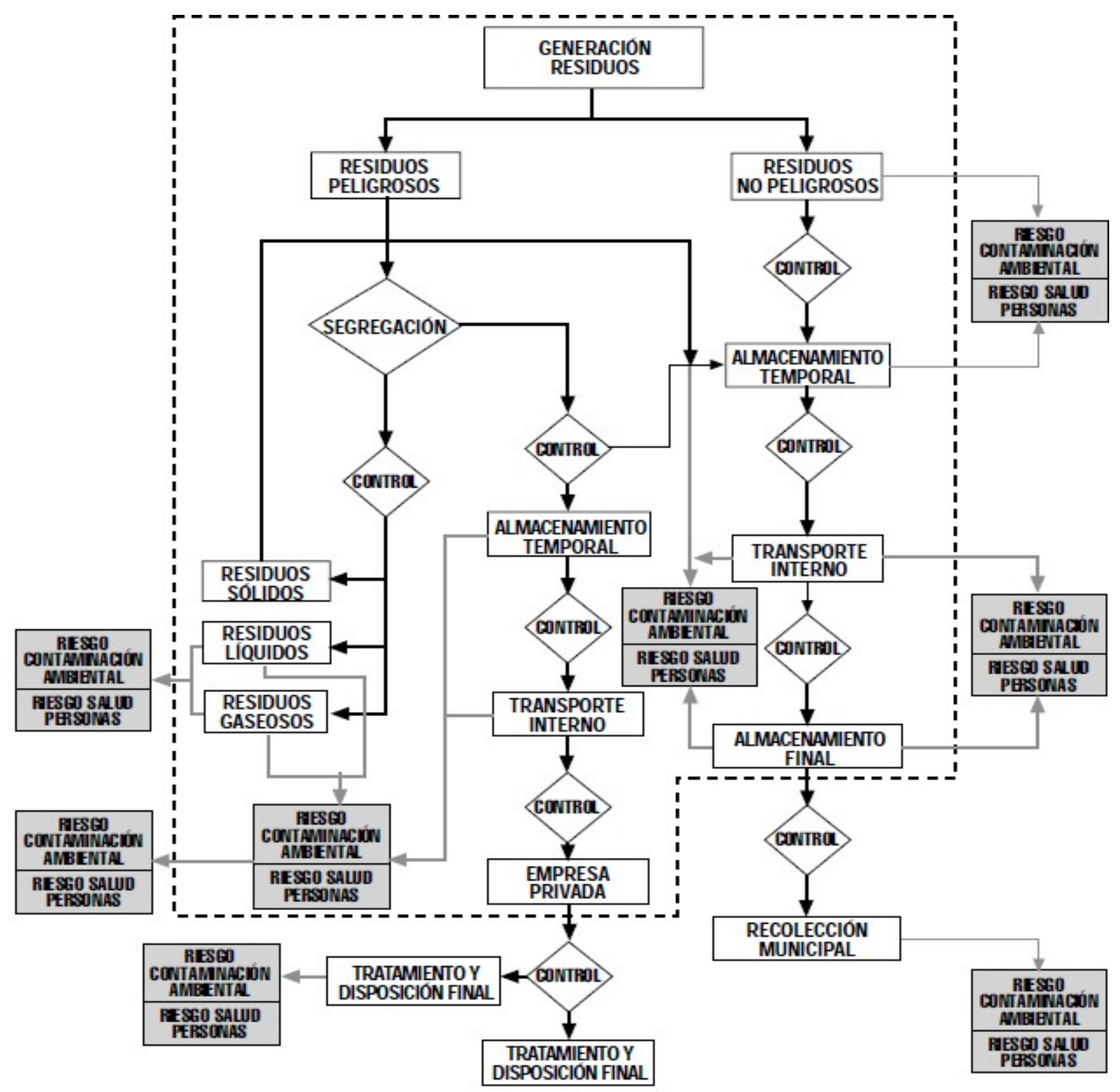

Figura 1. Modelo de manejo de los residuos hospitalarios

Fuente: (Neveu \& Matus, 2007) 
Todas estas variables son tomadas en cuenta en la atención sanitaria o la atención primaria de salud (APS). De aquí nace la importancia de la atención primaria como eje fundamental para el bienestar del paciente. Un sistema de salud basado en la APS se define como la operación de los sistemas de salud que hace el derecho a alcanzar el mayor nivel de salud posible su principal objetivo al tiempo que maximiza la solidaridad del sistema(Osorio \& Álvarez, 2013).Es parte de un sistema integrado de cuidados de la salud y del desarrollo económico-social de una sociedad, que involucra la cooperación con otros sectores para enfrentar los determinantes sociales de la salud y la promoción de la participación social(Giovanella, Almeida, Vega Romero, Oliveira, \& Tejerina Silva, 2015).

En el Ecuador, la APS esta bajo el amparo de la constitución de la republica y de la ley Orgánica de Salud a través del Ministerio de Salud Pública (MSP) el cual formulo un nuevo modelo de atención denominado Modelo de Atención Integral de Salud (MAIS). El MSP ofrece servicios de atención de salud a toda la población. El Ministerio de Inclusión Económica y Social y las municipalidades cuentan con programas y establecimientos de salud en los que también brindan atención a la población no asegurada, como también las instituciones de seguridad social cubren a la población asalariada afiliada (Lucio, Villacrés, \& Henríquez, 2019).

"Con ello, el MSP hizo suya la definición de salud como pilar del desarrollo humano sostenible, entendiendo que el desarrollo de las comunidades se enmarca en el cuidado de su salud y que, a través de un enfoque de promoción y prevención, es posible alcanzar el desarrollo individual y colectivo al que aspira el país: La salud y el enfoque de atención primaria en salud están en estrecha relación con la noción de desarrollo humano sustentable, ... plantea el desarrollo como bienestar y generación de oportunidades en el presente y para las generaciones futuras." (Espinosa, de la Torre, Acuña, \& Cadena, 2017, pág. 2)

Ahora, el sector de salud privado en el Ecuador está bajo el manto de las aseguradoras por medio de entidades con o sin fines de lucro. El sector privado comprende entidades con fines de lucro (hospitales, clínicas, dispensarios, consultorios, farmacias y empresas de medicina prepagada) y organizaciones no lucrativas de la sociedad civil y de servicio social(Lucio, Villacrés, \& Henríquez, 2019). 
Cristhian P. Triviño-Ibarra; Hernán D. Toro-Barrera; Hugo J. Saltos-Giler; Julio C. Cedeño-Quijije; María D. PárragaZambrano; Mercedes M. Vicuña-Castro

Tanto el sistema de salud en Ecuador público y privado se puede visualizar en la Figura 2 y la cantidad de entidades públicas y privadas, con o sin fines de lucro, dedicadas a la salud se detalla en la Figura 3.

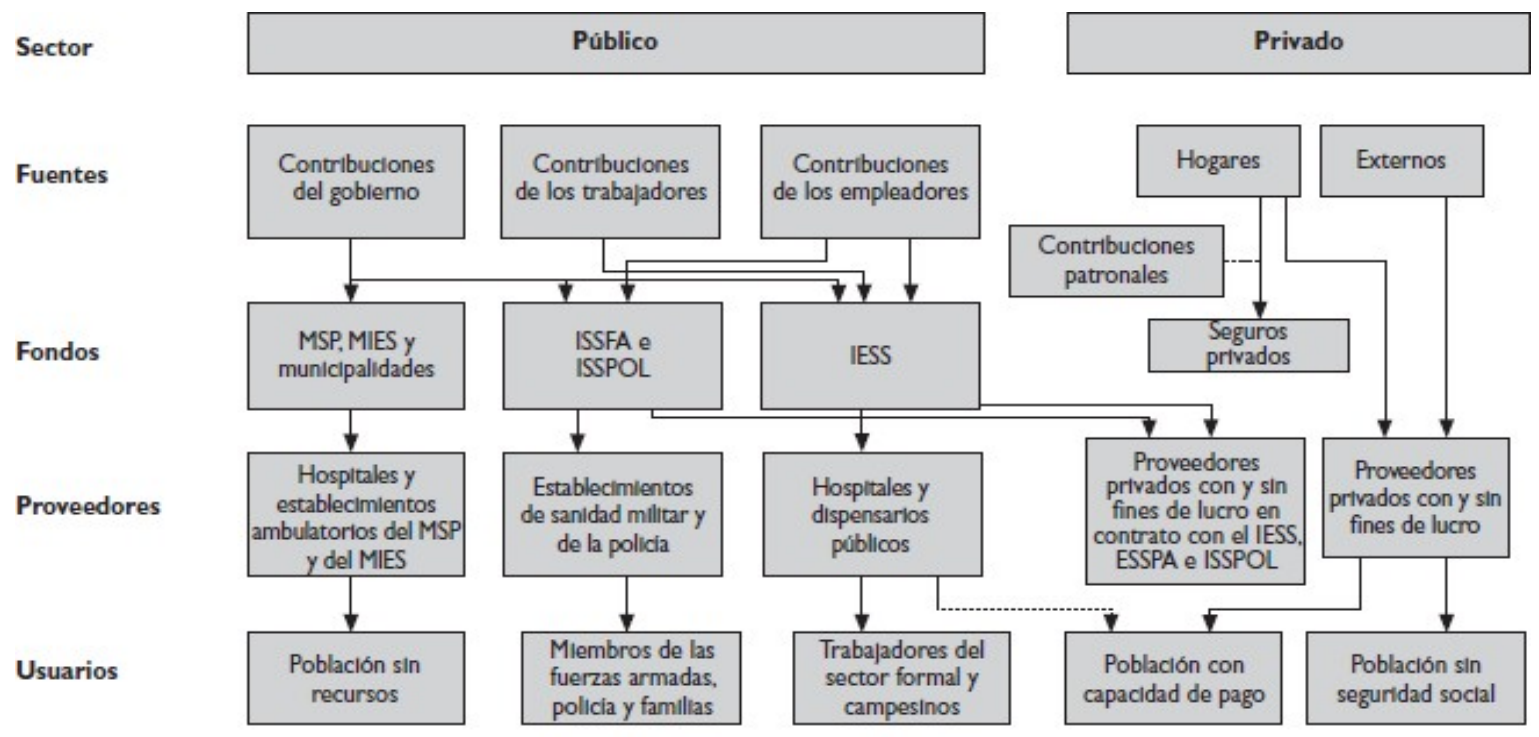

MSP: Ministerio de Salud Pública

MIES: Ministerio de Inclusión Económica y Social

ISSFA: Instituto de Seguridad Soclal de las Fuerzas Armadas
ISSPOL: Instituto de Seguridad Soclal de la Policia Nacional

IESS: Instituto Ecuatoriano de Seguridad Soclal

Figura 2. El sistema de salud en Ecuador

Fuente: (Lucio, Villacrés, \& Henríquez, 2019)

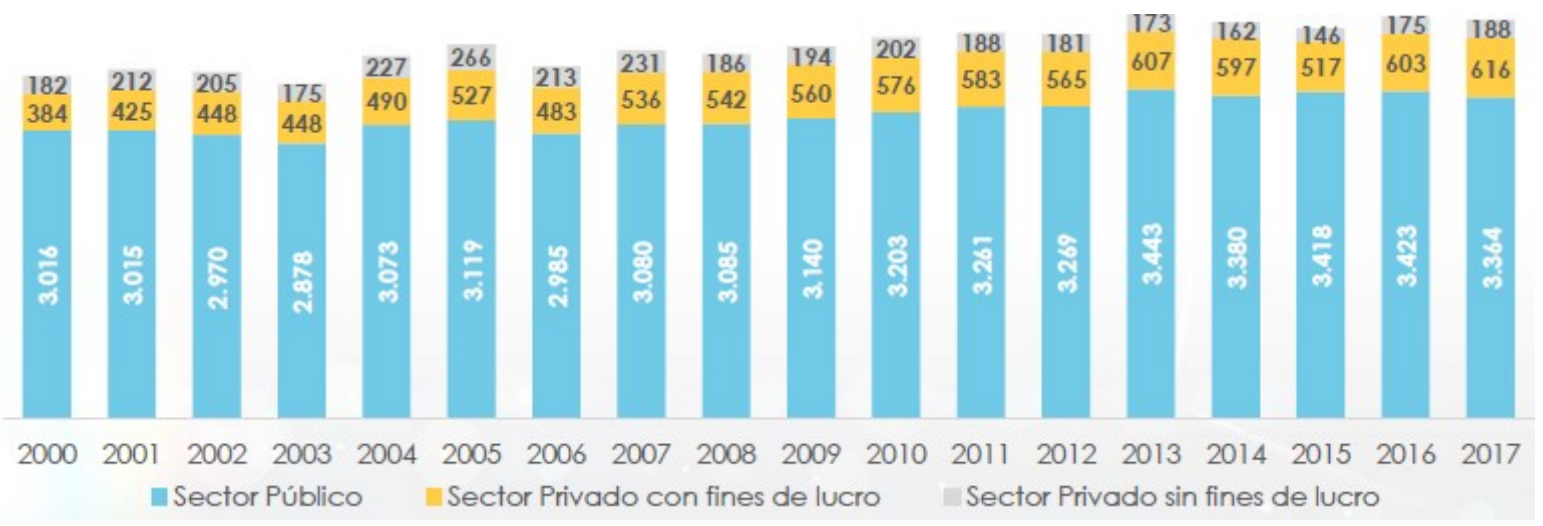

Figura 3. Establecimiento de salud por sector para el año 2017

Fuente: (INEC-RAS, 2019) 
Por lo tanto, esta investigación tiene como objetivo general analizar la importancia de la seguridad clínica hospitalaria para profesionales y pacientes. La metodología empleada se basó en investigaciones de tipo documental y bibliográfica.

\section{Método.}

La seguridad de los centros hospitalarios dependerá del conocimiento e identificación de los riesgos que puedan padecer los profesionales y pacientes que están dentro de estos sitios asistenciales. Poder controlar los errores y fallos que puedan suceder repercute directamente en la disminución d ellos riesgos ya que habrá pocos efectos adversos para ello. Poder concretar estos parámetros es necesario aplicar una metodología basada en investigaciones de tipo documental y bibliográfica a través de medio como textos, documentos y artículos científicos publicados disponibles en la web.

\section{Resultados.}

\section{Características de los servicios de salud}

Una de las características fundamentales para obtener o dar un mejor servicio en la atención al público es la formación para el trabajo. Esta es una mezcla entre educación, experiencia laboral yformación específica adquirida a lo largo de la vida, de ahí que las competencias sedefinen y se construyen en la práctica social y son una tarea conjunta entreempresas, trabajadores y educadores(Urbina Laza, 2011).

Esta formación es la principal base para una buena relación entre los principales actores en los sistemas de salud, los profesionales del área con los pacientes. Esta relación se ha deteriorado debido a que los profesionales no aplican la bioética y los pacientes responsabilizan a los medico y enfermeras por la situación patológica no esperada de la enfermedad. La responsabilidad médica surgida de su actuación profesional es unapreocupación de los médicos a nivel universal, ya que los reclamos porresponsabilidad médica inciden en la práctica asistencial, la relación médico-pacientey el gasto en salud(García, 2003).

Esto ha ayudado a la aparición de los pacientes activos, los cuales se han empoderado de su situación de salud, pero también al creciente cambio global en la era del acceso a la información y documentación. Además, la caracterización de la salud como un bien individual condiciona la 
Cristhian P. Triviño-Ibarra; Hernán D. Toro-Barrera; Hugo J. Saltos-Giler; Julio C. Cedeño-Quijije; María D. PárragaZambrano; Mercedes M. Vicuña-Castro

actitud de los pacientes sobre qué estrategias de cuidado consideran más adecuadas y cuáles son los objetivos terapéuticos que deben conseguir(Jovell, Rubio, Maldonado, \& Blancafort, 2006).

Con todos estos factores se establece un sistema de salud con características propias y con una serie de mecanismo que permiten el libre desarrollo y la practica del bien común. Es uno de los mejores mecanismos sociales para ayudar a disminuir la pobreza y mejorar la equidad en la sociedad evitando que los altos costos de la atención de salud se conviertan en un gasto directo adicional de los ciudadanos(Fuentes, 2016).

En Ecuador, el Sistema Nacional de Salud debe enfrentar una diversidad de retos y necesidades provenientes del perfil demográfico y epidemiológico.

"Los directivos de los hospitales pertenecientes al IESS para su gestión, deben disponer de herramientas que les permitan conocer el comportamiento de los parámetros demográficos, epidemiológicos y sociales de la población asignada a las instituciones que dirigen, así como indicadores de la actividad propia del hospital, de manera que una óptima gestión permita la adecuación de las labores asistenciales a las necesidades ciudadanas en el nivel local y en el desarrollo de mecanismos, que aseguren la adecuada calidad de la atención y la eficiencia en la utilización de los recursos”.(Batista Pereda, Yera Alós, Martínez Martín, Pérez Jiménez, \& Aranda Canosa, 2016, pág. 539)

Quien lleva la batuta dentro del ámbito de salud en el Ecuador es el MSP. Además, incluye fortalecer los servicios públicos de salud e incorporar recursos humanos cualificados y en número suficiente para brindar una atención de calidad y con calidez(Espinosa, de la Torre, Acuña, \& Cadena, 2017). La inclusión de profesionales de la salud dentro de las diferentes instituciones está reflejada en la Figura 4. Ahora, dentro del Sistema Nacional de Salud en Ecuador están los servicios de emergencia. Estos no han logrado consolidarse como un área especializada del sector salud y no cuentan con herramientas reguladoras (normativas detalladas y específicas para este tipo de servicios)(Santos \& Sierra, 2014). 


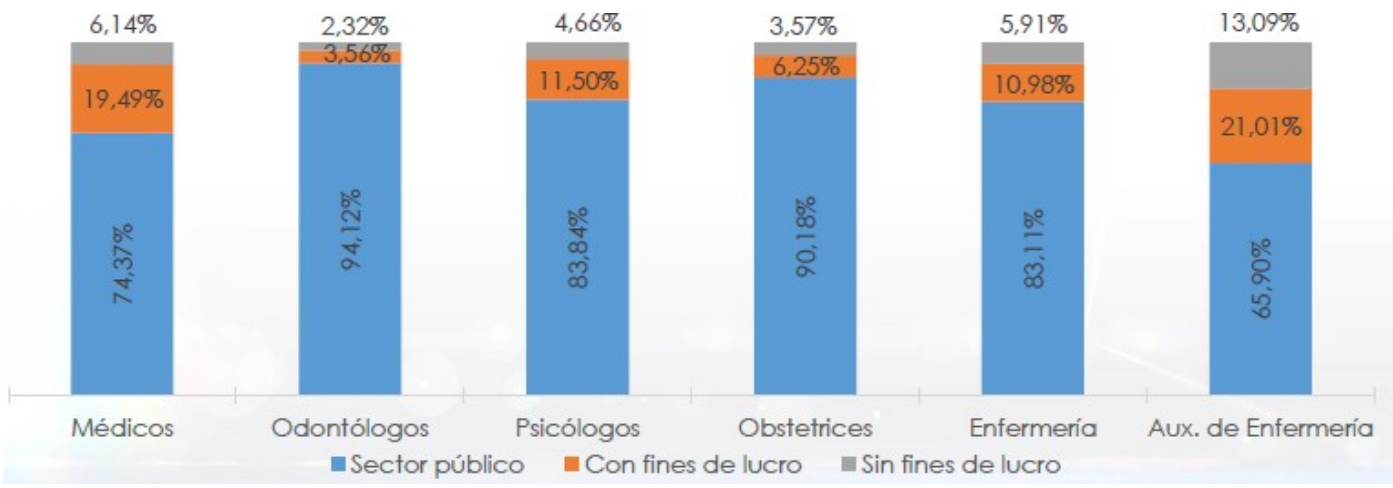

Figura 4. Profesionales de la salud por sector en Ecuador para el año 2017

Fuente: (INEC-RAS, 2019)

Por supuesto, toda esta fuerza movilizadora genera un gasto total de salud y que dependerá de la entidad que la suministre. Abarca la prestación de servicios de salud (preventivos y curativos), las actividades de planificación familiar, las actividades de nutrición y la asistencia de emergencia designadas para la salud, pero no incluye el suministro de agua y servicios sanitarios(Mancilla, 2013). Esto se puede observaren la Figura 5 donde se refleja el gasto de consumo final público y privado de la salud para el período 2007 al 2014. En la Figura 6 se detalla la distribución del gasto de consumo final total de productos característicos de la salud, Años 2007 y 2014.

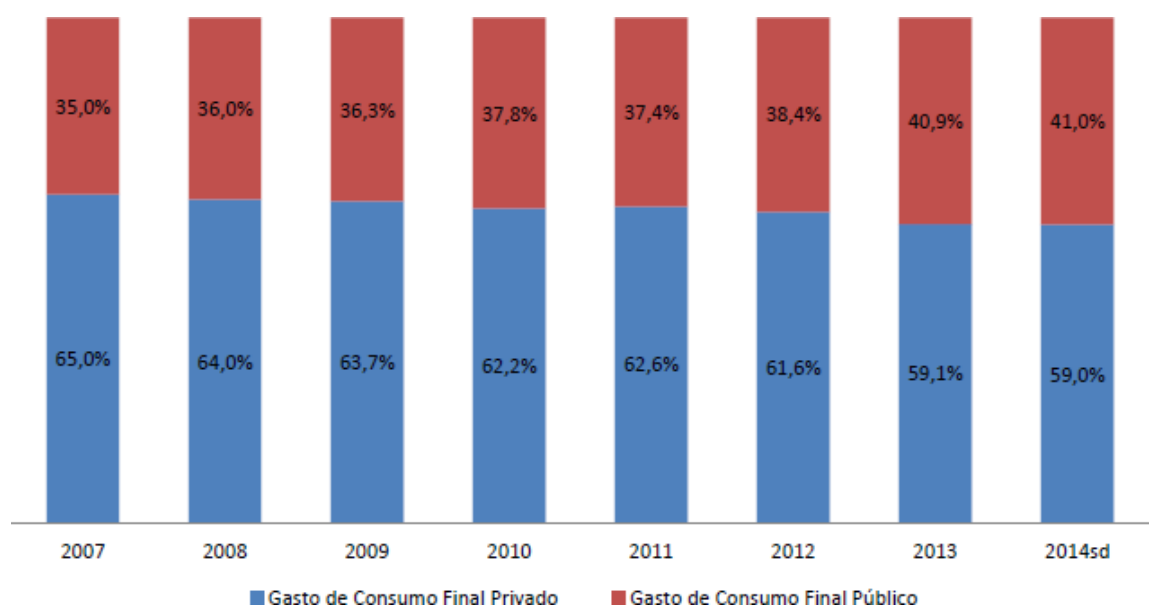

Figura 5. Gasto de consumo final público y privado de la salud para el período 2007 al 2014

Fuente: (INEC-CSS, 2019) 
Cristhian P. Triviño-Ibarra; Hernán D. Toro-Barrera; Hugo J. Saltos-Giler; Julio C. Cedeño-Quijije; María D. PárragaZambrano; Mercedes M. Vicuña-Castro

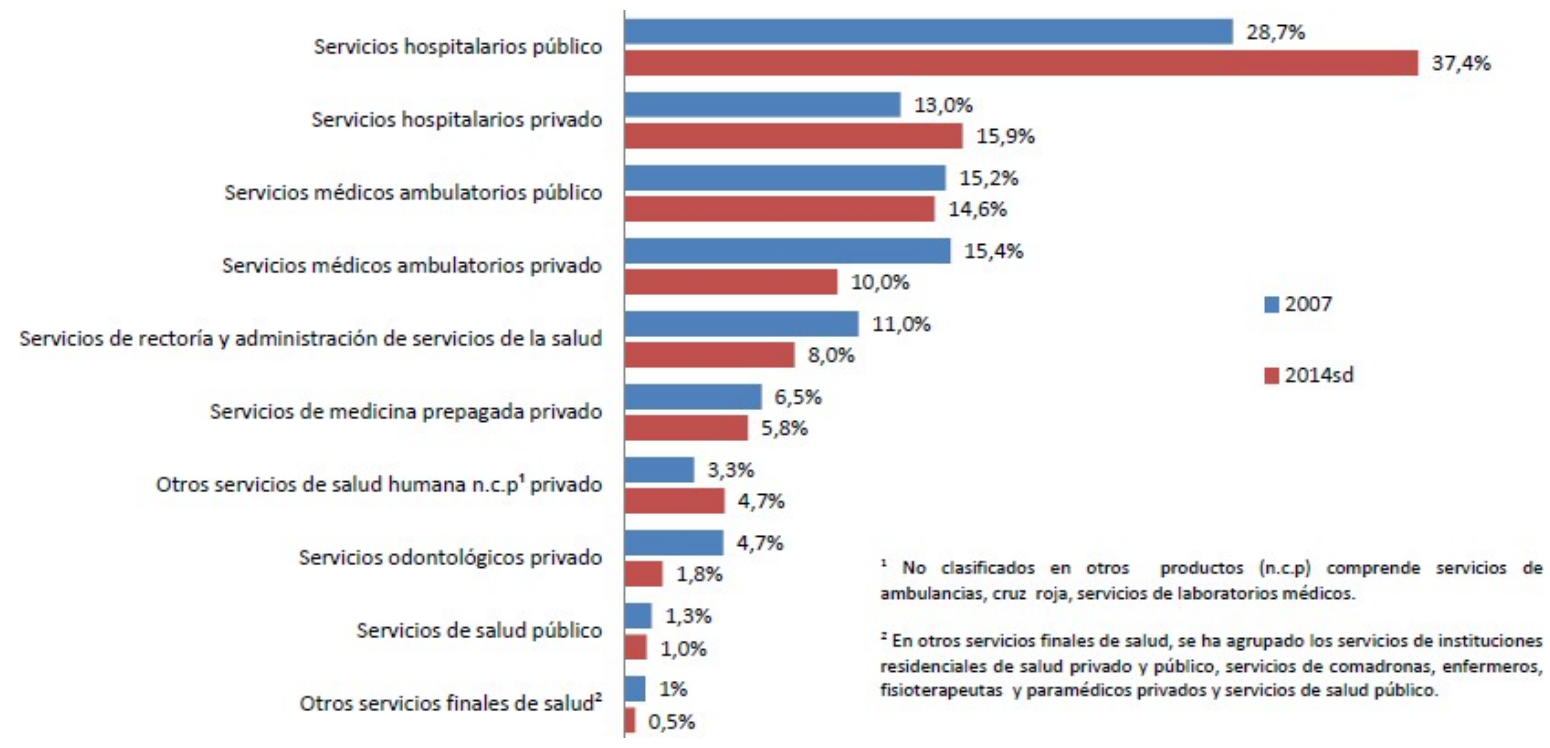

Figura 6. Distribución del gasto de consumo final total de productos característicos de la salud, Años 2007 y 2014

Fuente: (INEC-CSS, 2019)

Es por ello, que todos estos factores ayudan a mejorar los servicios de salud por lo cual introduce el término de la seguridad dentro de estos ámbitos. La medición de la cultura de la seguridad permite conocer la situación basal de la que partimos, así como priorizar las acciones de mejora y la repetición periódica de esta medida permite además analizar la efectividad de estas acciones y replantear estrategias(Egea, Vecina, \& Borras, 2011).

\section{La calidad dentro de los servicios de salud}

En Ecuador los principales beneficiarios de la salud están afiliados al IESS, es decir son personas asalariadas.

"Los beneficiarios del IESS están protegidos contra la contingencia de enfermedad por las siguientes prestaciones: asistencia médica, quirúrgica, dental y farmacológica integral. Estas prestaciones incluyen consulta externa, urgencias, medicina curativa, preventiva y de rehabilitación mediante la provisión de ortesis y prótesis; atención quirúrgica y hospitalización; atención médica domiciliaria; subsidio en dinero por enfermedad, y atención en unidades médicas ajenas al IESS”.(Lucio, Villacrés, \& Henríquez, 2019, pág. 182) 
Estas prestaciones se pueden detallar en la Figura 7 que muestra los ambientes físicos por región para el año 2017 en Ecuador.

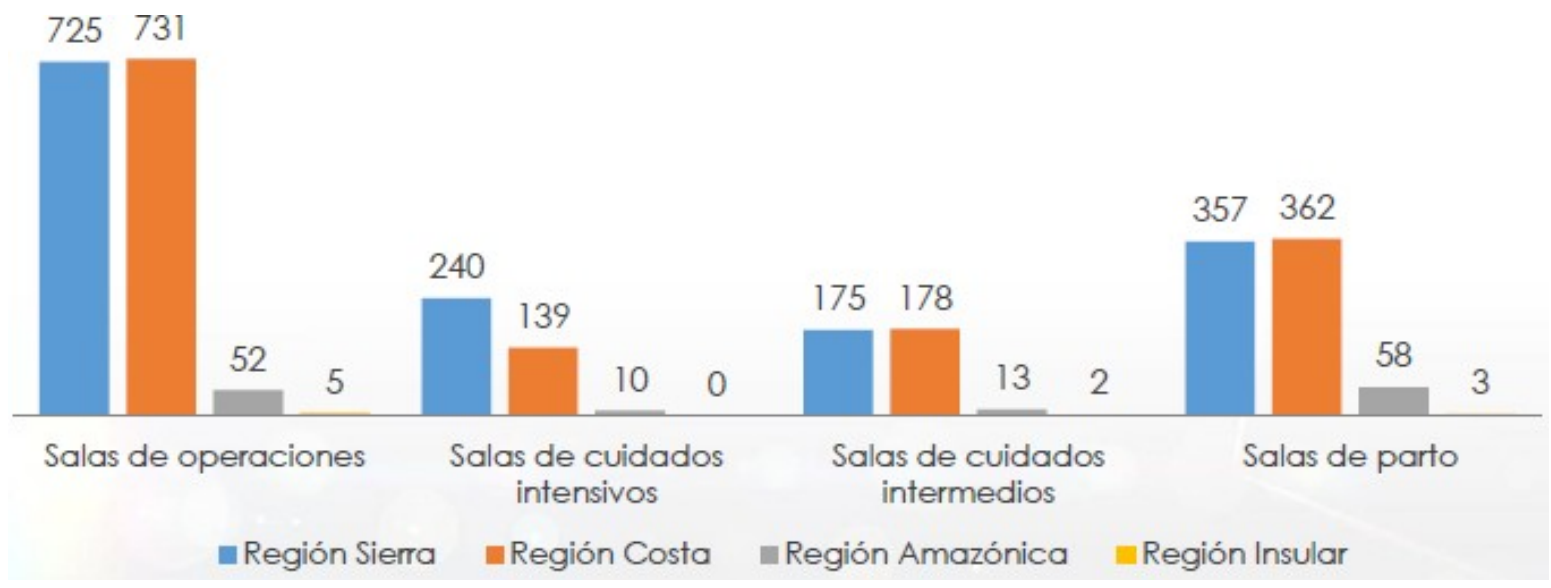

Figura 7. Los ambientes físicos por región para el año 2017 en Ecuador

Fuente: (INEC-RAS, 2019)

Estos ambientes físicos de salud están basados en parámetros de seguridad que involucran tanto a los profesionales como a los pacientes. Los profesionales están propensos a cometer errores en la aplicación de una técnica con lo cual pueden poner en peligro la salud y bienestar de quien la este ejecutando. Por esta razón, el elemento clave para prevenir las infecciones adquiridas, los incidentes y los accidentes es un personal preocupado por la seguridad y bien informado sobre la manera de reconocer y combatir los peligros que entraña su trabajo en ese entorno(Martel Aguirre, 2015). Las consecuencias de estos accidentes es producto de la fatiga y síndrome de Burnout que esta relacionado con el estrés laboral que involucra sus actividades y la exposición a diversos riesgos del trabajo en los ámbitos de la organización, el ambiente y área psico-social (Seguel \& Valenzuela, 2014).

También existe dificultad entre los profesionales y pacientes para poder establecer una relación acorde a la patología de la enfermedad basada en los buenos principios éticos y humanos. Dichas insatisfacciones están determinadas por una serie de factores contextuales, no directamente médicos, que emanan del contexto político, institucional y cultural, de manera que influyen y deben tenerse en cuenta(Almeida Vera \& Almeida Vera, 2014). Es por eso que se debe buscar estrategias que 
Cristhian P. Triviño-Ibarra; Hernán D. Toro-Barrera; Hugo J. Saltos-Giler; Julio C. Cedeño-Quijije; María D. PárragaZambrano; Mercedes M. Vicuña-Castro

permitan esta relación sea más factible y en pro del bienestar del paciente. Por tal motivo, en Ecuador el MAIS tiene un propósito:

"Es orientar el accionar integrado de los actores del Sistema Nacional de Salud hacia la garantía de los derechos en salud y el cumplimiento de las metas del Plan Nacional de Desarrollo para el Buen Vivir, al mejorar las condiciones de vida y salud de la población ecuatoriana a través de su implementación bajo los principios de la estrategia de la APS renovada, y transformar el enfoque biologicista hacia un enfoque integral en salud centrado en el cuidado y recuperación de la salud individual, familiar y comunitaria como un derecho humano".(Naranjo Ferregut, Delgado Cruz, Rodríguez Cruz, \& Sánchez Pérez, 2014, pág. 378)

Poder realizar estas acciones, enmarcadas en el plan del buen vivir se requiere una serie de etapas en las cuales destaca la seguridad dentro de los centros hospitalarios. Es aquí donde entra el termino bioseguridad.

"Es un término que ha sido utilizadopara de fin ir y congregar las normas decomportamiento y manejo preventivo, del personalde salud, frente a microorganismos potencialmenteinfecciosos, con el propósito de disminuir laprobabilidad de adquirir infecciones en el mediolaboral, haciendo énfasis en la PREVENCIÓN,mediante la asepsia y el aislamiento”.(Domínguez, 2015, pág. 26)

Estas normas de biodiversidad abarcan muchas áreas de los centros de salud ya que hay riesgos laborales. Estos riesgos se presentan al no hacer uso adecuado del material y equipo médicoquirúrgico donde debe aplicarse la técnica correcta y la más adecuada en el manejo tanto de equipos, medicamentos $\mathrm{y}$ en particular sobre muestras de pacientes con enfermedades infectocontagiosas(Plaza Moreira, 2012).

De aquí nace entonces la gestión de la calidad en estos centros de salud. Partiendo de la atención al paciente como de la seguridad laboral en el profesional de la salud. La gestión por la calidad del cuidado debe desarrollarse en el marco de una filosofía que asegure una atención integral, personalizada y libre de riesgo, que abarque la vulnerabilidad afectiva, física, mental y social del paciente en la búsqueda del bienestar(Jaramillo \& Pérez, 2017). 
Esta calidad dentro de los servicios de salud ha desarrollado metodologías variadas. Entre ellas esta la auditoría médica, conversatorios clínicos, comités de infección intrahospitalaria, comités de mortalidad; con el fin de queel mejoramiento de la calidad pueda evitar sufrimientos innecesarios, secuelas discapacitantes y altos costos en la atención de salud(Zapata López, 2014).

Por lo cual es importante poder definir este concepto de calidad dentro de los centros hospitalarios. Es el tipo de atención que maximiza el bienestar del paciente, después de tener en cuenta el balance de pérdidas y ganancias esperadas, contemplando el proceso de atención en todas sus partes(Galván, Moctezuma, Dolci, \& López, 2016). En la Figura 8 se puede observar el modelo para evaluar la calidad de los servicios de salud. 
Cristhian P. Triviño-Ibarra; Hernán D. Toro-Barrera; Hugo J. Saltos-Giler; Julio C. Cedeño-Quijije; María D. PárragaZambrano; Mercedes M. Vicuña-Castro

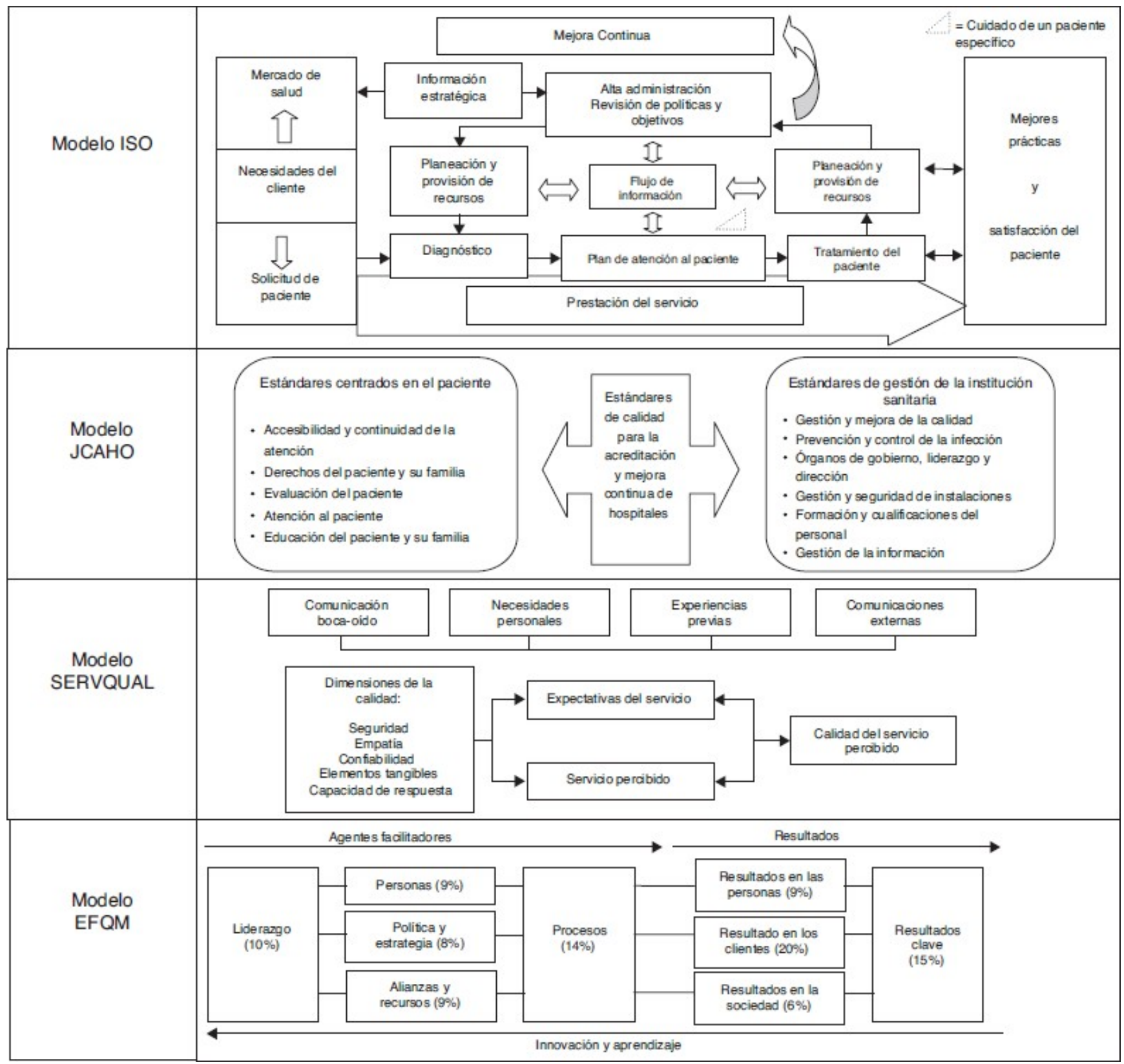

Figura 8. Modelo para evaluar la calidad de los servicios de salud

Fuente: (González, Melo, \& Limón, 2015)

Por supuesto la aplicación de la calidad en los centros de salud repercute en una inversión que debe hacer el estado para mejorar el servicio y así poder ofrecer el bienestar a la sociedad. Para ello, se debe trabajar fuertemente en 2 aspectos básicos para fortalecer el gasto público en salud: mantener un aumento del porcentaje del PIB invertido en salud, y alcanzar un fuerte sentido de 
institucionalidad para el cumplimiento de la Constitución vigente(Mancilla, 2013).esta relación entre el gasto de consumo final y el PIB del país esta reflejado en la Figura 9.

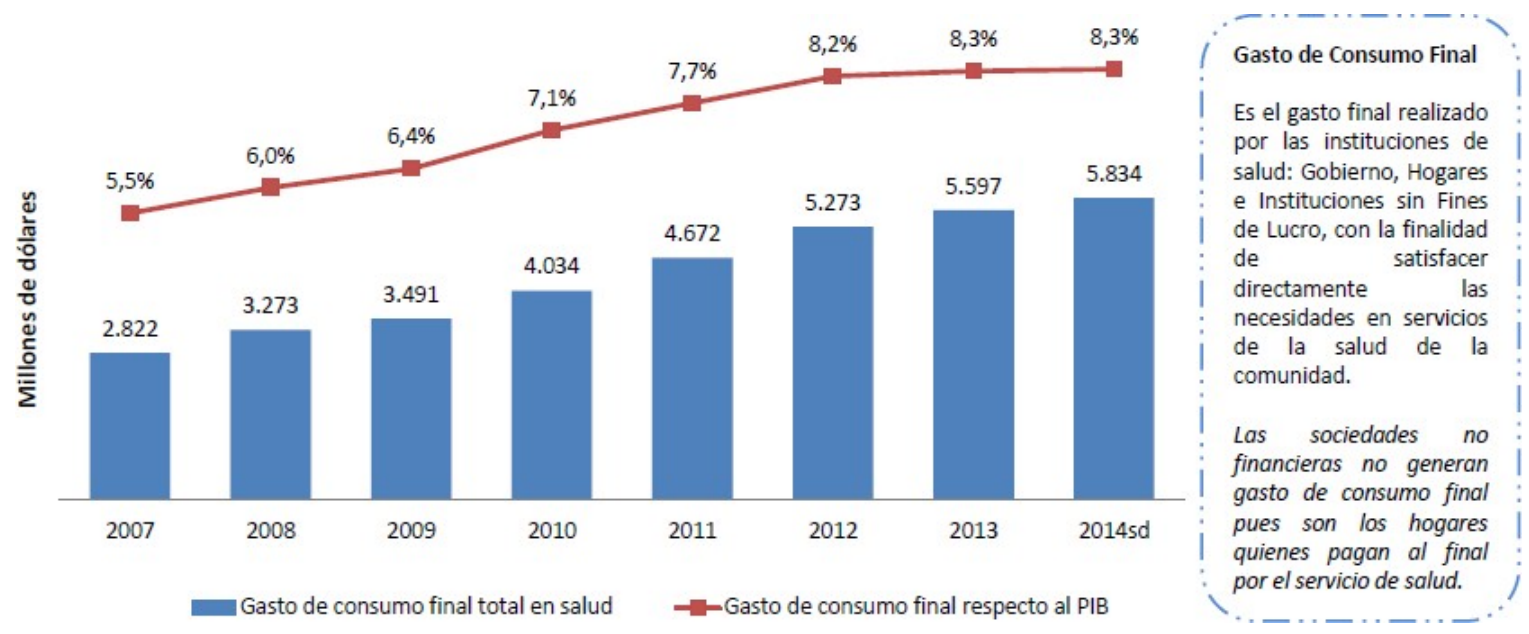

Figura 9. Gasto de consumo final de salud y su participación respecto al PIB. Período 2007- 2014

Fuente: (INEC-CSS, 2019)

\section{El riesgo en los centros de salud}

Una de las consecuencias que esta presente dentro de los centros hospitalarios es el riesgo de sufrir algún accidente o de padecer otra enfermedad. De aquí radica la importancia de poder conocer el riesgo en ambientes laborables. Es la probabilidad que un incidente o evento adverso ocurra(MSP, 2015).El riesgo de una actividad puede tener dos componentes: la posibilidad o probabilidad de que un resultado negativo ocurra y el tamaño de ese resultado; por lo tanto, mientras mayor sea la probabilidad y la pérdida potencial, mayor será el riesgo(Echemendía Tocabens, 2011).

La existencia de riesgo es común en cualquier lugar de trabajo, lo importante es poder tener precaución en la realización de las tareas. El riesgo está sometido a dos factores.

"Estos se clasifican, en general, en factores de amenaza y factores de vulnerabilidad. Una amenaza refiere a la posibilidad de la ocurrencia de un evento físico que puede causar algún tipo de daño a la sociedad. La vulnerabilidad se refiere a una serie de características diferenciadas de la sociedad, o subconjuntos de la misma, que le predisponen a sufrir daños frente al impacto de un evento físico externo, y que dificultan su posterior recuperación”.(Lavell, 2001, pág. 2) 
Cristhian P. Triviño-Ibarra; Hernán D. Toro-Barrera; Hugo J. Saltos-Giler; Julio C. Cedeño-Quijije; María D. PárragaZambrano; Mercedes M. Vicuña-Castro

Ahora es necesario poder comprender el riesgo dentro de las instalaciones de salud, tanto para los profesionales como para los pacientes. Un factor detonante en el riesgo son los errores que cometen las personas o técnicas involucradas. Los errores deben analizarse desde la perspectiva de que se producen porque existen fallos en los componentes del sistema (systemapproach)y no por incompetencia o fallos de los individuos (personapproach), como ha sido el enfoque tradicional hasta ahora(Otero López, 2003).

Otro aspecto para que ocurran riesgos dentro de los lugares asistenciales es el saneamiento dentro de estos recintos. Los pisos y paredes sucias, filtraciones en los techos, baños inadecuados, entre otros son factores que inciden en la precipitación del riesgo en el ambiente de trabajo. El saneamiento básico es una de las medidas fundamentales para minimizar y/o controlar el riesgo potencial de infecciones intrahospitalarias(Borges, 1998).

Esto permite que ocurra otras enfermedades fuera del cuadro patologico por lo cual un paciente ingresa a un centro de salud. La infección nosocomial (IN) es un claro ejemplo de estas evenatualidades productos del riesgo. Según la Organización Mundial de la Salud, es la que se presenta en un paciente cuando es internado en un hospital u otro establecimiento de atención de salud, la cual no se había manifestado ni estaba en período de incubación al ingreso(Vázquez Belizón, González Aguilera, González Pompa, García, \& Lázaro, 2013).

Es por ello que se deben aplicar ciertas estrategias o acciones que reduzcan el riesgo. Esta se puede definir como todas aquellas intervenciones que se hacen en estructuras o en procesos de atención en salud para minimizar la probabilidad de ocurrencia de un incidente o evento adverso(MSP, 2015).

Entonces se debe aplicar una metodología que impida la formación de un evento o suceso adverso. Esto se puede visualizar en la Figura 10.

"La aparición de un suceso adverso es la parte más visible del iceberg de los riesgos asistenciales $\mathrm{y}$, con frecuencia, es la última secuencia de un proceso que ha comenzado con anterioridad, en el que han influido múltiples factores humanos y relacionados con los distintitos niveles organizativos del sistema sanitario”.(Aibar \& Aranaz, 2003, pág. 202) 


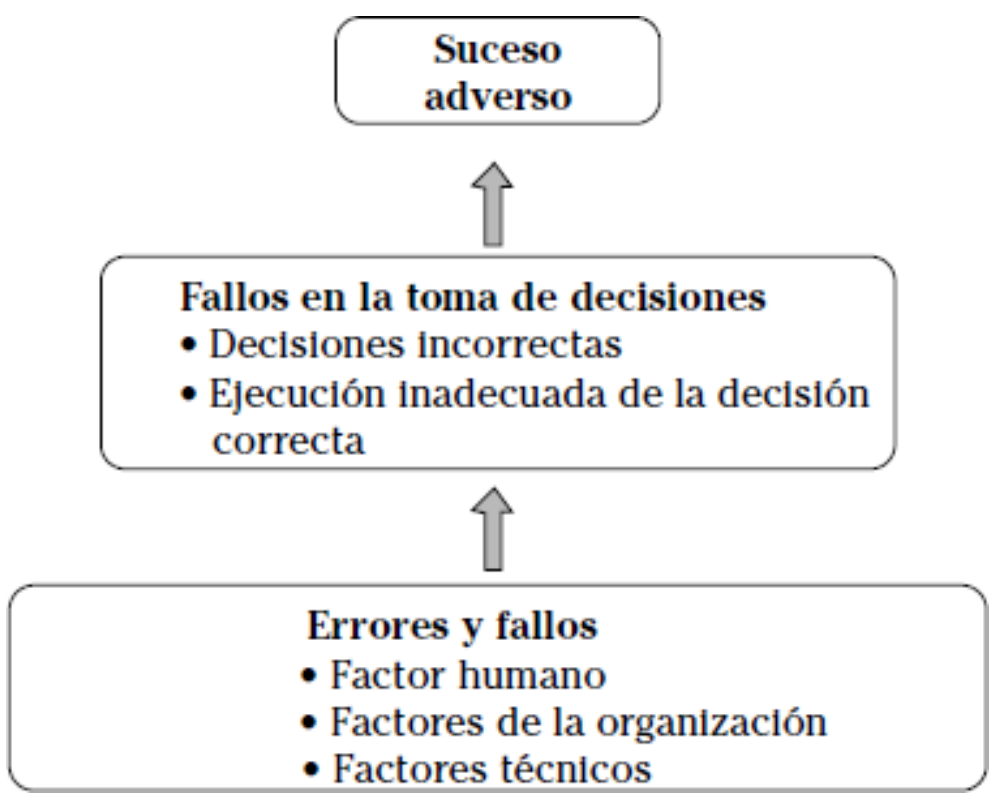

Figura 10. El iceberg de los sucesos adversos

Fuente: (Aibar \& Aranaz, 2003)

Los errores son los agentes catalizadores de los eventos o sucesos adversos por lo que se debe buscar maneras para disminuir tales fallos. Estos eventos o sucesos se dividen en dos grupos los cuales estarán definidos bajo los términos de responsabilidad ética, penal, civil y administrativa. Estos son los eventos adversos prevenible y no prevenible. El evento adverso no prevenible es la complicación que no puede ser prevenida dado el estado actual del conocimiento; el evento adverso prevenible es el mal resultado de la atención que puede ser prevenido con el estado del conocimiento(Urbina Laza, 2011).

En muchos centros hospitalarios estos sucesos no ocurren con frecuencia, pero si destaca que proceden de la condición en la relación de los profesionales con el paciente. Por lo cual es necesario poder transformar esta tendencia en un factor de confianza. Para ello resulta imprescindible contarcon la participación de los ciudadanos en todos los aspectosde la asistencia sanitaria a través de los mecanismosdispuestos para ellos (consejos de salud, asociacionesde pacientes, etc.)(Aranaz, y otros, 2006). 
Cristhian P. Triviño-Ibarra; Hernán D. Toro-Barrera; Hugo J. Saltos-Giler; Julio C. Cedeño-Quijije; María D. PárragaZambrano; Mercedes M. Vicuña-Castro

Ahora también es necesario poder determinar cuales son los factores de riesgo de los profesionales de la salud dentro de los centros hospitalarios. Estos riesgos surgen de la exposición constante a una serie de agentes aparentemente no tan lesivos tales como sustancias químicas inhalatorios, ruido, la manipulación de objetos punzo cortantes y otros elementos(Calabrese, 2005). Hay otros riesgos complementarios como la el empuje o arrastre de camillas y el levantamiento de objetos incrementa la posibilidad de trastornos musculo esqueléticos(Castañeda Lara, 2012). En la Tabla 1 se detalla los riesgos biológicos, físicos, químicos y estructurales que pueden sufrir los profesionales de la salud.

\section{Tabla 1. Riesgos que pueden padecer los profesionales de la salud en los centros hospitalarios}

- Riesgos por agentes biológicos $\rightarrow$ fluido corporal infectado con gérmenes patógenos como sangre, derivados de la sangre, líquido cerebroespinal, amniótico, pleural, pericardio, peritoneal, sinovial, exudados inflamatorios, etc.

- Riesgos por agentes ambientales $\rightarrow$ ventilación, temperatura, eliminación de residuos de gases anestésicos, látex, antisépticos, solventes, sustancias esterilizadoras, de desinfección, etc.

- Riesgos por agentes físicos $\rightarrow$ ruidos y vibraciones, radiaciones ionizantes, radiaciones no ionizantes (láser), etc.

- Riesgos por agentes estructurales $\rightarrow$ son aquellos vinculados a carga térmica, ventilación/circulación de aire, iluminación/fatiga visual, aparatos eléctricos de alto y bajo voltaje, incendios y gases comprimidos (balones), etc.

- Los riesgos biológicos $\rightarrow$ son aquellos causados por organismos vivos, generalmente microscópicos, que pueden ser virales, bacterianos o por hongos.

Fuente: (Calabrese, 2005)

\section{Discusión y conclusiones.}

Una de las características que se deben tomar en cuentan dentro de los lugares de trabajo es la seguridad y el ambiente laboral que tengan los trabajadores. La seguridad es la técnica que requiere 
de una evaluación objetiva por parte del individuo y la organización con el fin de poder disminuir los accidentes cuando se esta en presencia de algún peligro que perjudique la salud y bienestar de la persona. Esta evaluación objetiva va a depender de la experiencia, conocimientos y focalización de la persona que esta ejecutando una tarea en específico. Es por ello, que debe cumplir con los objetivos, visión y misión de la organización. Dentro de los centros hospitalarios no escapa la seguridad ya que hay factores que puedan catalizar algún evento que permita desmejorar la salud de los trabajadores y pacientes de dicho lugar. Para ello es necesario poder aplicar una cultura de seguridad que permita romper los viejos paradigmas dentro de estos centros de salud y poder realizar procesos de gestión de la seguridad. Es necesario poder aplicar estos métodos de gestión para poder disminuir la factibilidad de riesgos presentes en ese lugar.

Indudablemente que una buena seguridad dentro de los centros hospitalarios hace que la atención sanitaria sea más eficiente y por lo cual disminuya los errores en sucesos que puedan disminuir la calidad y bienestar de las personas presentes dentro de este lugar, sea profesional de la salud o paciente. Es por ello, que la atención sanitaria juega un papel importante para el desarrollo y evolución positiva dentro de la patología del paciente. Uno de los factores que puedan incrementar este parámetro es la relación entre profesional-paciente. Una relación más estrecha hace que el paciente pueda recuperarse y hacer que su tratamiento cumpla con los objetivos dentro de la terapia de recuperación. Otra característica de esta relación es que los mismos pacientes han aplicado el empowerment y se han convertido en el principal motivador para superar sus propios problemas de salud. Esta atención sanitaria esta manifestada a través d ellos diferentes programas y políticas que implementa el estado para el bienestar de la sociedad, llamándolo atención primaria de salud. Estos buscan a través de varios medios, ofrecer una calidad de servicio a los pacientes con el fin de poder aliviar los problemas de salud que puedan padecer.

Nuevamente, una mejor atención primaria de salud genera una calidad en los servicios hospitalarios. Esta calidad debe seguir los estándares internacionales que buscan maximizar el bienestar d ellos pacientes y a su vez generar dentro de los profesionales de salud una mayor confianza y satisfacción en las tareas realizadas. Por eso, es necesario la aplicación de la calidad, no solo en la búsqueda de dicho bienestar sino también en la seguridad de los presentes en estos lugares. Esta seguridad permite la identificación y control de los riesgos que puedan suceder y que puedan manifestarse en contra de la salud de quien pueda percibirlo. 
Cristhian P. Triviño-Ibarra; Hernán D. Toro-Barrera; Hugo J. Saltos-Giler; Julio C. Cedeño-Quijije; María D. PárragaZambrano; Mercedes M. Vicuña-Castro

Es así como el conocimiento e identificación del riesgo forma parte fundamental de la seguridad dentro de los centros hospitalarios. Tanto los pacientes como los profesionales están propensos a sufrir riesgos, pero con estos parámetros las consecuencias de estos eventos adversos disminuyen ya que disminuyen también los errores y fallos presentes en estos lugares. Por lo tanto, la seguridad dentro de los centros de salud en un deber de todos aquellos que forman parte, desde la gerencia hasta los pacientes y familiares que acuden a estos recintos.

\section{Bibliografia.}

Aibar, C., \& Aranaz, J. (2003). ¿ Pueden evitarse los sucesos adversos relacionados con la atención hospitalaria? In Anales del sistema sanitario de Navarra (Vol. 26, No. 2). Gobierno de Navarra. Departamento de Salud., 195-209.

Almeida Vera, L., \& Almeida Vera, L. (2014). Fundamentación del modelo de gestión intercultural ecuatoriana en la atención primaria de salud. Medisan, 18(8), 1170-1183.

Aranaz, J. M., Aibar, C., Galán, A., Limón, R., Requena, J., Álvarez, E. E., \& Gea, M. (2006). La asistencia sanitaria como factor de riesgo: los efectos adversos ligados a la práctica clínica. Gaceta sanitaria, 20, 41-47.

Batista Pereda, Y., Yera Alós, I. B., Martínez Martín, M. I., Pérez Jiménez, D., \& Aranda Canosa, S. (2016). El análisis de la situación de salud hospitalaria: herramienta para la gestión de hospitales del Instituto Ecuatoriano de Seguridad Social. Revista Médica Electrónica, 38(4), 530-542.

Betancourt, O. (1999). Salud y Seguridad en el Trabajo. Quito - Ecuador: OPS, OMS-FUNSAD.

Borges, A. (1998). Personal de Enfermería: Condiciones de trabajo de alto riesgo. Salud de los Trabajadores, 6(2), 113-119.

Calabrese, G. (2005). Riesgos biológicos y ambientales. Revista Argentina de Anestesiología, 235, 235-252.

Castañeda Lara, A. G. (2012). Evaluación de los riesgos relativos a la manipulación de pacientes en la unidad del centro quirúrgico del Hospital Provincial Docente Ambato. Quito - Ecuador: Trabajo de Grado - UNIVERSIDAD SAN FRANCISCO DE QUITO.

Domínguez, Y. A. (2015). Evaluación de conocimientos y practicas sobre bioseguridad, Hospital IESS Ibarra, Agosto 2014. Revista Desafios, 9(2), 25-39.

Echemendía Tocabens, B. (2011). Definiciones acerca del riesgo y sus implicaciones. Revista Cubana de Higiene y Epidemiología, 49(3), 470-481.

Egea, F. R., Vecina, S. T., \& Borras, M. R. (2011). Cultura de seguridad del paciente en los servicios de urgencias: resultados de su evaluación en 30 hospitales del Sistema Nacional de Salud español. Emergencias, 23, 356-364. 
Espinosa, V., de la Torre, D., Acuña, C., \& Cadena, C. (2017). Los recursos humanos en salud según el nuevo modelo de atención en Ecuador. Revista Panamericana de Salud Pública, 41, e52., 1-5.

Fuentes, R. C. (2016). El Sistema Nacional de Salud de Ecuador: Estudiarlo para mejorarlo. Alternativas, 17(2), 60-64.

Galván, H. R., Moctezuma, J. M., Dolci, G. F., \& López, D. O. (2016). De la idea al concepto en la calidad en los servicios de salud. Revista Conamed, 17(4), 172-175.

García, M. (2003). La responsabilidad civil del médico en el Ecuador. Quito - Ecuador: Recuperado de http://www. bioetica. org. ec/articulos/articulo_responsabilidad_civil. pdf.

Giménez-Júlveza, T., Hernández-García, I., Aibar-Remón, C., Gutiérrez-Cíac, I., \& FebrelBordejéd, M. (2017). Cultura de la seguridad del paciente en directivos y gestores de un servicio de salud. Gac Sanit., 31(5), 423-426.

Giovanella, L., Almeida, P. F., Vega Romero, R., Oliveira, S., \& Tejerina Silva, H. (2015). Panorama de la Atención Primaria de Salud en Suramérica: concepciones, componentes y desafíos. Saúde em Debate, 39, 300-322.

González, I. B., Melo, N. A., \& Limón, M. L. (2015). El clima organizacional y su relación con la calidad de los servicios públicos de salud: diseño de un modelo teórico. Estudios gerenciales, 31(134), 8-19.

INEC-CSS. (30 de Mayo de 2019). Cuentas satelite de los servicios de salud (CSS). 2007-2014. Obtenido de http://www.ecuadorencifras.gob.ec/documentos/webinec/Estadisticas_Economicas/Cuentas_Satelite/Cuentas_Satelite_Salud/1_Presentacion_CS S_2007-2014.pdf

INEC-RAS. (30 de Mayo de 2019). Regístro estadístico de recursos y actividades de salud. Obtenido de http://www.ecuadorencifras.gob.ec/documentos/webinec/Estadisticas_Sociales/Recursos_Actividades_de_Salud/RAS_2017/Principales_Resulta dos_\%28RAS\%29.pdf

Jaramillo, M. N., \& Pérez, J. I. (2017). Calidad del cuidado enfermero en un centro quirúrgico: Experiencia en un hospital de Ibarra, Ecuador. Enfermería Investiga: Investigación, Vinculación, Docencia y Gestión, 2(4), 132-136.

Jovell, A. J., Rubio, M. D., Maldonado, L. F., \& Blancafort, S. (2006). Nuevo rol del paciente en el sistema sanitario. Atención primaria, 38(4), 234-237.

Lavell, A. (2001). Sobre la gestión del riesgo: apuntes hacia una definición. Biblioteca Virtual en Salud de Desastres-OPS, 4, 1-22.

León Román, C. A. (2006). La seguridad del paciente, una responsabilidad en el acto del cuidado. Revista Cubana de enfermería, 22(3), 1-7. 
Cristhian P. Triviño-Ibarra; Hernán D. Toro-Barrera; Hugo J. Saltos-Giler; Julio C. Cedeño-Quijije; María D. PárragaZambrano; Mercedes M. Vicuña-Castro

Lucio, R., Villacrés, N., \& Henríquez, R. (30 de Mayo de 2019). Sistema de salud de Ecuador. Obtenido de salud pública de méxico / vol. 53, suplemento 2 de 2011: https://www.scielosp.org/pdf/spm/2011.v53suppl2/s177-s187/es

Mancilla, J. C. (2013). Gasto público en salud en el Ecuador. Medicina, 18(1), 53-60.

Martel Aguirre, P. (2015). Aplicación de normas de bioseguridad del profesional de enfermería en centro quirúrgico. Lima - Perú: Trabajo de Grado - Universidad de San Martín de Porras, FACULTAD DE OBSTETRICIA Y ENFERMERÍA.

MSP. (2015). Norma Técnica de Seguridad del Paciente. Cuenca - Ecuador: Coordinación Zonal de Salud 6, Hospital Vicente Corral Moscoso.

Muiño Míguez, A., Jiménez Muñoz, A. B., Pinilla Llorente, B., Durán García, M. E., Cabrera Aguilar, F. J., \& Rodríguez Pérez, M. P. (2007). Seguridad del paciente. In Anales de medicina interna. Vol. 24, No. 12, 602-606.

Naranjo Ferregut, J. A., Delgado Cruz, A., Rodríguez Cruz, R., \& Sánchez Pérez, Y. (2014). Consideraciones sobre el modelo de atención integral de salud del Ecuador. Revista Cubana de Medicina General Integral, 30(3), 375-381.

Natura, F., \& Zabala, L. M. (1997). Manual para el Manejo de Desechos en Establecimientos de Salud. Quito, Ecuador. Fundación Natura, Comité Interinstitucional para el Manejo de Desechos Hospitalarios.

Neveu, A., \& Matus, P. (2007). Residuos hospitalarios peligrosos en un centro de alta complejidad. Revista médica de Chile, 135(7), 885-895.

Osorio, A. G., \& Álvarez, C. V. (2013). La Atención Primaria de Salud: desafíos para su implementación en América Latina. Atención primaria, 45(7), 384-392.

Otero López, M. J. (2003). Errores de medicación y gestión de riesgos. Revista española de salud pública, 77, 527-540.

Plaza Moreira, J. (2012). Fortalecimiento de las normas de bioseguridad en hospitales y centros de salud del Ecuador para la obtención de un modelo sanitario seguro. Guayaquil - Ecuador: Trabajo de Grado - Universidad de Guayaquil.

Quinto-Mosquera, Y., Pérez, L. M., \& Arias, J. A. (2013). Conocimientos y prácticas de los trabajadores de un hospital sobre el manejo de residuos hospitalarios, Chocó, Colombia, 2012. Revista Médicas UIS, 26(1)., 9-20.

Ramos Domínguez, B. N. (2005). Calidad de la atención de salud: Error médico y seguridad del paciente. Revista Cubana de Salud Pública, 31(3), 239-244.

Revuelta, J. F., \& Mingorance, M. B. (2011). Conceptos básicos sobre seguridad clínica: definición e importancia del problema. Revista Enfermería del Trabajo, 1(4), 221-228. 
Santos, P. S., \& Sierra, J. P. (2014). Los servicios de emergencias médicas en el Ecuador: una tarea pendiente. Revista de la Facultad de Ciencias Médicas de la Universidad de Cuenca, 32(3), 58-69.

Seguel, F., \& Valenzuela, S. (2014). Relación entre la fatiga laboral y el síndrome burnout en personal de enfermería de centros hospitalarios. Enfermería universitaria, 11(4) , 119-127.

Urbina Laza, O. (2011). Competencias de Enfermería para la seguridad del paciente. Revista Cubana de Enfermería, 27(3), 239-247.

Vázquez Belizón, Y. E., González Aguilera, J. C., González Pompa, J. A., García, S., \& Lázaro, A. (2013). Factores de riesgo de infección intrahospitalaria en pacientes ingresados en unidades de cuidados intensivos. Medisan, 17(8), 3068-3076.

Zapata López, C. D. (2014). Sistemas de gestión de calidad y aplicación en los servicios hospitalarios de salud pública del Ecuador. Quito - Ecuador: Trabajo de Grado UNIVERSIDAD SAN FRANCISCO DE QUITO.

Zula Majín, N. (2015). Seguridad de los pacientes durante la atención de enfermería en el servicio de emergencias del Hospital de Los Valles de la ciudad de Quito, de julio a diciembre 2014. Guayaquil, Ecuador: Trabajo de Grado - Universidad de Guayaquil. Facultad de Ciencias Médicas. 